\title{
Ancient Pbx-Hox signatures define hundreds of vertebrate developmental enhancers
}

\author{
Hugo J Parker ${ }^{1}$, Paul Piccinelli ${ }^{1}$, Tatjana Sauka-Spengler ${ }^{2}$, Marianne Bronner ${ }^{3}$ and Greg Elgar ${ }^{1 *}$
}

\begin{abstract}
Background: Gene regulation through cis-regulatory elements plays a crucial role in development and disease. A major aim of the post-genomic era is to be able to read the function of cis-regulatory elements through scrutiny of their DNA sequence. Whilst comparative genomics approaches have identified thousands of putative regulatory elements, our knowledge of their mechanism of action is poor and very little progress has been made in systematically de-coding them.

Results: Here, we identify ancient functional signatures within vertebrate conserved non-coding elements (CNEs) through a combination of phylogenetic footprinting and functional assay, using genomic sequence from the sea lamprey as a reference. We uncover a striking enrichment within vertebrate CNEs for conserved binding-site motifs of the Pbx-Hox hetero-dimer. We further show that these predict reporter gene expression in a segment specific manner in the hindbrain and pharyngeal arches during zebrafish development.

Conclusions: These findings evoke an evolutionary scenario in which many CNEs evolved early in the vertebrate lineage to co-ordinate Hox-dependent gene-regulatory interactions that pattern the vertebrate head. In a broader context, our evolutionary analyses reveal that CNEs are composed of tightly linked transcription-factor binding-sites (TFBSs), which can be systematically identified through phylogenetic footprinting approaches. By placing a large number of ancient vertebrate CNEs into a developmental context, our findings promise to have a significant impact on efforts toward de-coding gene-regulatory elements that underlie vertebrate development, and will facilitate building general models of regulatory element evolution.
\end{abstract}

Keywords: Gene regulation, enhancer code, sea lamprey, Hox genes, embryogenesis

\section{Background}

Cis-regulatory elements play an essential role in the precise co-ordination of vertebrate development as illustrated by the increasing number of examples where mutations in such sequences lead to developmental malformations [1-3]. One of the major challenges in modern biology is the deciphering of the regulatory language, syntax and grammar, encoded in the genome, that directs spatio-temporally restricted gene expression. To achieve this requires the identification and functional characterisation of cis-regulatory elements, followed by the deconvolution of the TFBSs therein.

Cis-regulatory elements can be predicted by sequence conservation analysis, as tight clusters of functional

\footnotetext{
* Correspondence: gelgar@nimr.mrc.ac.uk

'Division of Systems Biology, MRC National Institute for Medical Research,

The Ridgeway, Mill Hill, London NW7 1AA, UK

Full list of author information is available at the end of the article
}

TFBSs can be under strong evolutionary constraint [4-7]. Alternatively, targeted approaches involving chromatin immunoprecipitation (ChIP) can be used to identify binding-events between specific transcription factors and DNA [8-10]. These are complementary approaches, as ChIP analyses are restricted to identifying regulatory regions that are targets of selected TFs at the particular time-points chosen for the analysis, whilst sequence conservation can identify elements irrespective of the TFs that bind to them or the developmental time-points at which they act. Sequence conservation can also provide evidence for ancient gene regulatory network (GRN) interactions that are shared between species. Comparative approaches applied to vertebrate genomes have identified a set of putative regulatory elements showing extreme conservation across mammals (Ultraconserved elements [11]), as well as Conserved Noncoding Elements (CNEs) shared between mammals and
C Biomed Central

() 2011 Parker et al; licensee BioMed Central Ltd. This is an Open Access article distributed under the terms of the Creative Commons Attribution License (http://creativecommons.org/licenses/by/2.0), which permits unrestricted use, distribution, and reproduction in any medium, provided the original work is properly cited. 
fishes $[6,12,13]$. These elements are clustered around developmental genes [6,12] and a large proportion of CNEs that have been tested in transgenic assays drive spatially restricted reporter gene expression in mouse or zebrafish embryos $[6,7,14]$. Furthermore, a number of CNEs have been shown to have roles in developmental diseases $[1,15]$. Despite their high sequence conservation between vertebrates, only a minute fraction of CNEs can be traced back to invertebrate chordates [16]. Thus, CNEs represent a set of cis-regulatory elements that are likely to be fundamental during development of the vertebrate body plan and comprise a valuable resource for deciphering the genomic regulatory code for vertebrate development.

Phylogenetic footprinting has been successfully implemented to identify TFBSs that play key roles in the action of individual CNEs [17-19]. However, there have been very few studies seeking to place large numbers of deeply conserved CNEs into a developmental context through using this approach [20,21]. Furthermore, despite progress having been made identifying key sequence motifs within vertebrate promoter elements [22], ancient CNEs have remained somewhat recalcitrant to systematic motif-identification algorithms, despite some elegant targeted approaches. Within non-coding elements conserved amongst mammals, a large number of long motifs [12-22 nucleotides) [23] and some shorter motifs [24] have been identified as overrepresented. However, the majority of these were not matched to any known factors, nor linked to any patterns of enhancer activity, so the biological significance of these motifs is hard to interpret. Recently, an elegant study used a classifier algorithm to identify sequence motifs predictive of heart enhancer activity in mammalian CNEs [25]. However, mammalian CNEs represent a set of sequences that only partially overlap with the more ancient mammal-fish CNEs and it is not clear to what extent they are functionally and mechanistically alike. Studies seeking to identify motifs that contribute to tissue-specific expression of deeply conserved vertebrate CNEs have discovered novel motifs associated with forebrain enhancer activity [7,21]. As part of a large-scale project to characterise the in-vivo enhancer activity of CNEs, Pennacchio et al. [7] used four human-fugu CNEs that drove forebrain reporter expression in mouse embryos to identify 6 enriched 5 bp-long sequence motifs. 23 elements enriched for these motifs were tested for enhancer activity, of which 4 were found to drive forebrain expression - an enrichment for this expression domain compared to the original enhancer set. Li et al. [21] characterised 13 CNEs driving forebrain reporter expression in zebrafish embryos, identifying 5 enriched motifs of $6 \mathrm{bp}$ and demonstrating that these sequences contributed to forebrain enhancer activity. These investigations go some way towards providing a developmental context for the CNEs with those motifs, but this is somewhat limited by the factors that bind to them remaining uncharacterised. Whilst it is unclear to what extent ancient vertebrate CNEs are composed of 'conventional', previously characterised TFBSs, candidate motif search approaches have provided evidence that mammalian UCEs are enriched for known TFBS motifs [26] and that ancient vertebrate CNEs associated with genes involved in CNS development show enrichment for Oct and Sox motifs [20]. The success of these isolated studies hints that it may be possible to systematically identify functional TFBSs within CNEs by phylogenetic footprinting.

The sea lamprey (Petromyzon marinus) hails from an anciently diverging jawless vertebrate lineage, the agnathans, which split from the jawed vertebrate lineage 550-650 million years ago [27]. We have previously found a significant number of CNEs that are conserved between lamprey and jawed vertebrates [28]. We predicted that the relatively low sequence identity between the lamprey and jawed-vertebrate homologous elements would facilitate the identification of conserved TFBS motifs within them. In addition, characterisation of these motifs could illuminate ancient GRN interactions common to all vertebrates. Thus, we sought to identify TFBS motifs in CNEs by performing phylogenetic footprinting, using the lamprey elements as a guide.

Here we identify deeply conserved TFBS motifs for the Pbx-Hox heterodimer within a cluster of CNEs associated with the meis 2 gene. We use in-silico analyses to demonstrate that jawed vertebrate CNEs and other sets of conserved vertebrate enhancers are highly enriched in $\mathrm{Pbx}$-Hox motifs. Using reporter assays in zebrafish and lamprey embryos, we show that these motifs correlate with enhancer function in the hindbrain and pharyngeal arches. These results represent a further step toward decoding vertebrate CNEs, allowing a large proportion of them to be more firmly placed into a developmental context and revealing ancient gene regulatory network interactions for hindbrain patterning that are shared across vertebrates. Finally, our findings enable us to hypothesise an evolutionary scenario regarding the role of many CNEs in the evolution of the vertebrate hindbrain and the branchial region of the head.

\section{Results}

A set of meis2 CNEs drive expression in the hindbrain and cranial ganglia in zebrafish and lamprey embryos

We previously identified a genomic region, downstream of the developmental gene meis2, containing a number of CNEs that are conserved between jawed vertebrates and lamprey [28] (Additional File 1). We grouped these CNEs into five separate elements (Additional File 2) for 
functional testing in a zebrafish tol2 reporter assay [29]. Four of these elements drive discreet and complementary patterns of reporter expression in the hindbrain of zebrafish embryos, with homologous zebrafish and lamprey elements driving highly similar expression patterns (Figure 1). These patterns of reporter expression are consistent with the endogenous expression of meis 2 in the hindbrain $[30,31]$, where Meis proteins play a crucial patterning role by interacting with Hox and $\mathrm{Pbx}$ transcription factors $[32,33]$. Lamprey and zebrafish CNE 3285 elements both drive GFP expression in the cranial ganglia and primary neurons of the hindbrain and spinal cord. CNE 3288 elements of zebrafish and lamprey drive GFP in neurons of the hindbrain posterior to rhombomere $4(\mathrm{r} 4)$, as shown by comparison to RFP expression in $\mathrm{r} 3$ and $\mathrm{r} 5$ in a transgenic line containing RFP under

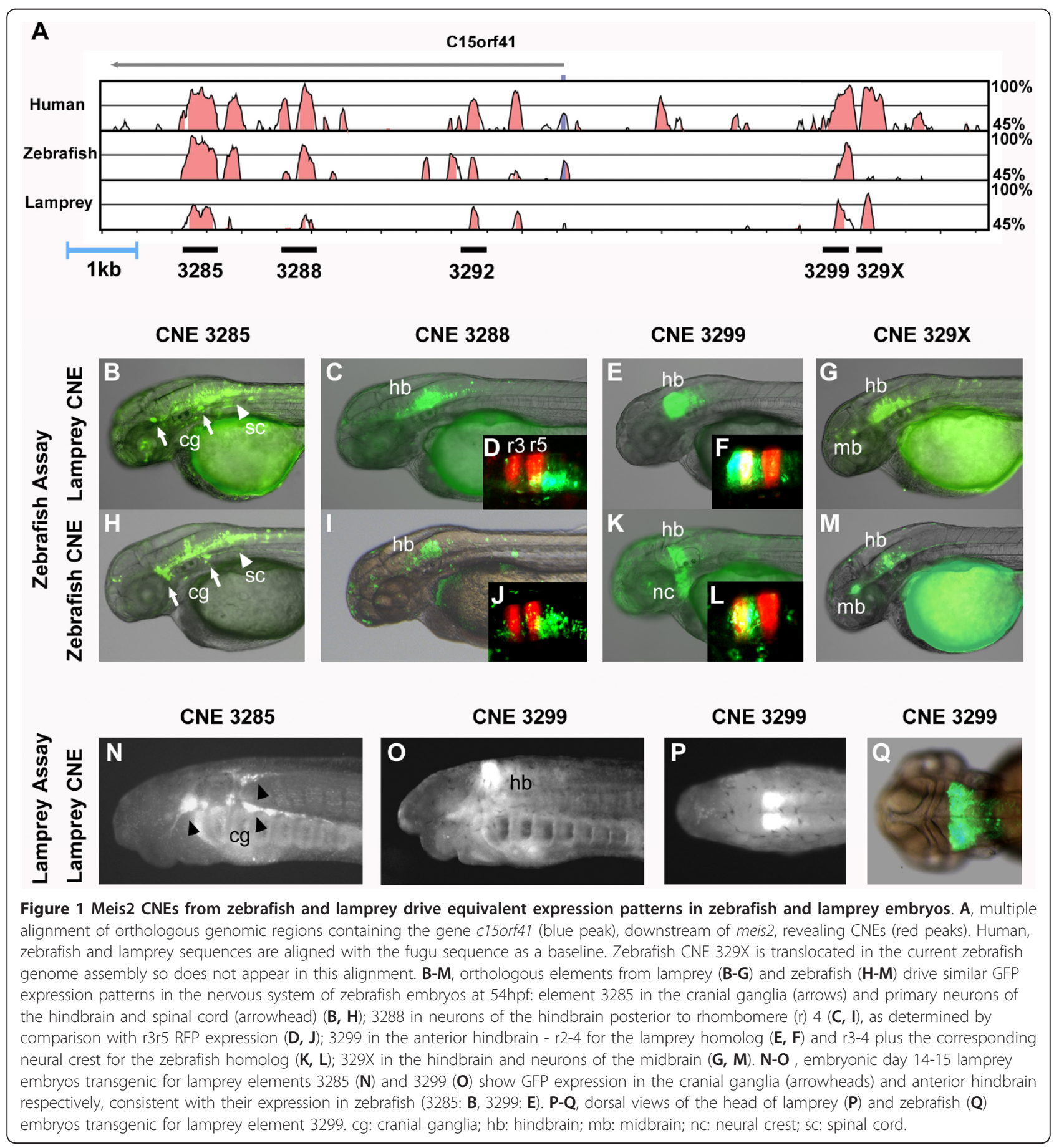


the control of a krox20 regulatory element [34]. CNE 3299 elements up-regulate GFP in the anterior hindbrain - r2-4 for the lamprey homolog and r3-4 plus neural crest migrating into the hyoid pharyngeal arch for the zebrafish homolog. CNE 329X of lamprey and zebrafish both drive GFP expression in the anterior hindbrain and neurons of the midbrain.

We have developed a parallel reporter assay in lamprey embryos (in submission) to assess the functional conservation of CNEs across vertebrates. Using this assay, we have tested lamprey CNEs 3285 and 3299 for enhancer activity during lamprey embryogenesis. In lamprey embryos, CNE 3285 drives GFP expression in the cranial ganglia and CNE 3299 in the anterior hindbrain (Figure $1)$. Thus, for both of these elements, the pattern of reporter expression driven in lamprey embryos is almost identical to the pattern driven in zebrafish embryos (Figure 1). This provides compelling evidence that these CNEs are part of a gene-regulatory network for hindbrain patterning that is conserved across all vertebrates.

\section{Some meis2 CNEs contain deeply conserved Pbx-Hox TFBS motifs}

Because of its clear and specific expression pattern in the hindbrain of zebrafish and lamprey embryos, we chose element 3299 as a starting point for the identification of putative transcription-factor binding-sites by phylogenetic footprinting. A number of studies have documented a role for the anterior Hox proteins in regulating rhombomere-specific gene expression by binding as hetero-dimers and -trimers with the TALE-class homeodomain proteins $\mathrm{Pbx}$ and Meis [17,18,35,36]. These complexes bind to characteristic binding-sites composed of partially overlapping Pbx-Hox half-sites, frequently in conjunction with a distal Meis/Prep site $[17,18,35,36]$. In some cases it has been shown that the pbx-hox motif is both necessary and sufficient for highly specific patterns of reporter expression, for instance for activity of a mouse hoxb1 enhancer in $\mathrm{r} 4$ in the mouse hindbrain [17] and for $\mathrm{r} 4$ and pharyngeal arch activity of a mouse hoxb2 enhancer [36].

We identified two Pbx-Hox motifs within CNE 3299, conforming to the TGATNNAT consensus [37,38], that are conserved across all sequenced vertebrate genomes, each closely associated with conserved Meis motifs (TGACAG/A) [39] (Figure 2). In the zebrafish sequence, the first pair of Pbx-Hox and Meis motifs is also preceded by a Pbx-Meis motif (TGATTGACAG/A) [39]. We verified the essential nature of these motifs for rhombomere-specific activity of the enhancer through mutagenesis of the zebrafish element followed by reporter assay (Methods). Mutating the first cluster of motifs (sub1) resulted in a loss of the neural crest expression of the wild type enhancer and less anteriorly restricted expression in the hindbrain compared to the wild type element (Figure 2b, c). Mutation of the second Pbx-Hox and Meis motif cluster abrogated reporter expression by this enhancer altogether, whilst a construct in which both motif clusters were mutated (sub12) also drove no GFP expression. Interestingly, CNEs 3285, 3288 and $329 \mathrm{X}$ were also found to harbour conserved $\mathrm{Pbx}-\mathrm{Hox}$ and Meis motifs. Together, the expression patterns of these elements in the hindbrain $(3285,3288,3299$, $329 \mathrm{X})$, as well as in the pharyngeal arch neural crest (3299), suggests that these motifs may represent a common feature of CNEs that drive segment-specific expression patterns in the vertebrate hindbrain and pharyngeal arches.

\section{Pbx-Hox motifs are enriched in CNEs and in other sets of conserved vertebrate enhancers}

In order to address how widespread Pbx-Hox motifs are across conserved vertebrate enhancers, we performed a systematic scan for these motifs in vertebrate CNEs. We searched for instances of the canonical Pbx-Hox motif, TGATNNAT, that are completely conserved across CNE multiple sequence alignments. In a set of 246 alignments of CNEs between human, zebrafish, fugu

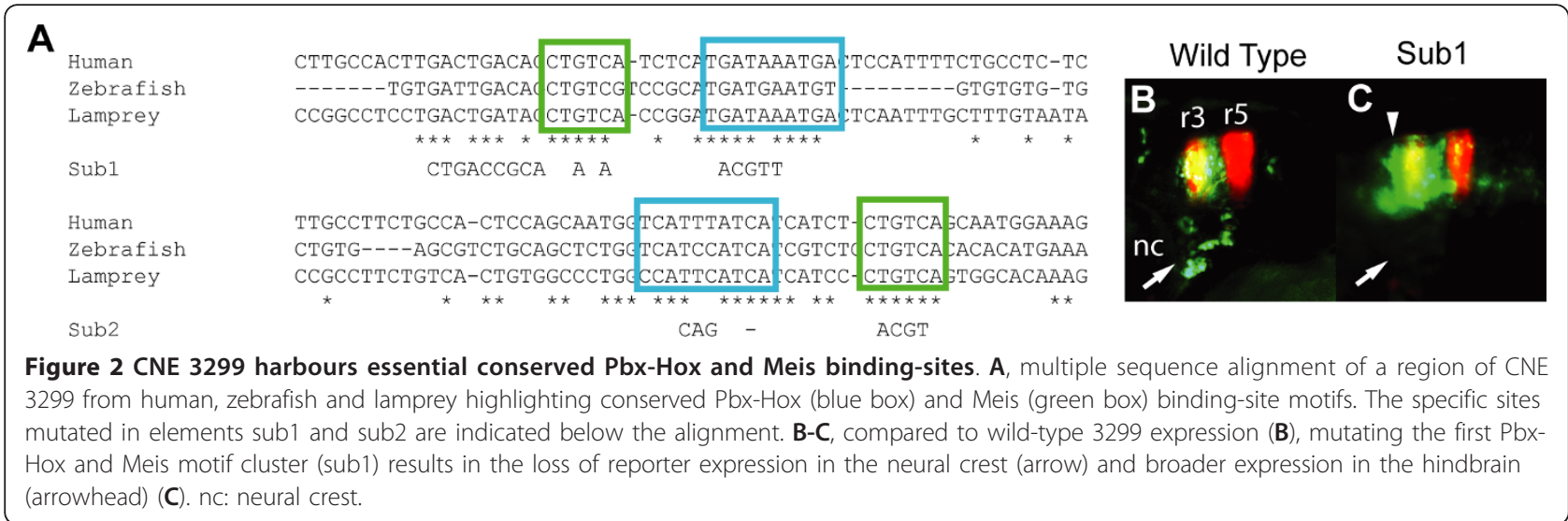


and lamprey (Methods), we identified 61 conserved motifs, representing 22 fold enrichment over shuffled alignments (Methods and Additional File 3). Furthermore, in a set of 4259 gnathostome CNE alignments (of human, fugu and zebrafish sequences), 712 conserved motifs were identified; a 9 fold enrichment compared to shuffled alignment controls.

Further analysis of $\mathrm{Pbx}$-Hox motifs in the gnathostome set reveals a paucity of cytosines at variable positions 5 and 6 (Figure 3). This is a feature of characterised $\mathrm{Pbx}$ Hox binding-sites, where $\mathrm{T}, \mathrm{A}$ or $\mathrm{G}$ at these positions contribute to determining the Hox specificity of the binding site $[38,40,41]$. Furthermore, positions 9 and 10 , immediately 3 ' to the canonical Pbx-Hox motif, show strong bias towards G/T and A/G respectively, thereby defining a more stringent TGATNNATKR (KR) consensus motif that is also consistent with previously characterised Pbx-Hox binding-sites $[17,18,37,38]$ (Figure 3). Further analysis of the lamprey and gnathostome CNE alignment sets results in even stronger enrichment for this 'KR' motif (Additional File 3).

We complemented our 'bottom-up' search for PbxHox motifs in CNEs with a 'top-down' de novo motif search using the tool Cis-Finder [42]. Strikingly, one of the top-scoring predicted motifs identified by Cis-Finder matches our consensus KR motif for a set of 6, 693 human sequences from the CONDOR CNE database [43] ('CONDOR CNEs') (Figure 3, Methods). The KR motif occurs 562 times in this CNE set, representing a highly significant enrichment over shuffled versions of the motif $\left(\mathrm{p}=5.7 \times 10^{-5}\right)$, and when compared to control genomic regions and the entire human genome (Table 1, Methods and Additional File 4). Interestingly, the Meis motif is also significantly enriched in the CONDOR CNE set $\left(\mathrm{p}=1.0 \times 10^{-4}\right)$ (Additional File 5). We then examined the distribution of $K R$ motifs in other sets of evolutionarily conserved non-coding sequences. The VISTA Enhancer Browser (EB) [44] contains over 1300 human sequences, around half of which drive reporter gene expression in mouse embryos at day 11.5. There is a significant enrichment for the KR motif $(p=0.0033)$ across the entire dataset compared with shuffled versions despite the fact that some of the sequences in EB are not deeply conserved (Table 1). Finally, we analysed a large set of deeply conserved human CNEs identified through comparison with the cartilaginous chimera, Callorhinchus milii [13], and once again found significant enrichment for the KR motif $\left(\mathrm{p}=6.2 \times 10^{-5}\right)($ Table 1 - 'Shark CNEs').

\section{Pbx-Hox motifs are associated with hindbrain and pharyngeal arch CNE enhancer function}

Next, we tested whether Pbx-Hox motifs within CNEs associate with segment-specific reporter expression in the hindbrain and pharyngeal arches. To do this, we assayed 21 zebrafish CNEs containing conserved $\mathrm{Pbx}-$ Hox motifs for reporter expression in zebrafish. All of these CNEs are conserved across gnathostomes, with 11 also identifiable in lamprey (Additional File 2). Elements were chosen to represent a range of different genes from the lamprey and gnathostome CNE sets. 12 of these 21 elements consistently up-regulate patterns of reporter expression, comprised of 8 from the lamprey set and 4 from the gnathostome set. It should be noted that some of the elements from which no consistent expression patterns were obtained may act as enhancers in-vivo, but not in our transient transgenic reporter assay, possibly due to being taken out of their genomic context. Remarkably, 11 of the 12 GFP-expressing elements (91.6\%) drive expression either in the hindbrain, pharyngeal arches or both, with one element expressing in the trunk musculature (Figure 4). In support of the hypothesis that these elements are directly regulated by specific Hox proteins, which have segmentally-restricted expression patterns, the majority of the elements expressing in the hindbrain do so in particular rhombomeres, as shown by comparison with r3r5 RFP expression (Figure 4). Hindbrain reporter expression driven by these elements is often further restricted dorso-ventrally (e.g. Nkx6-1_4281), medio-laterally (e.g. Pax2_217) and temporally (e.g. Tshz3_43509).

We next examined functional data from the VISTA Enhancer Browser (EB). Compared to shuffled motifs, the KR motif was found to be significantly enriched in those elements annotated as hindbrain positive as well as those positive for either hindbrain, branchial arch or cranial nerve expression (Table 1). Investigating those EB elements that overlap CNEs from the CONDOR set, we found significant enrichment for the KR motif in those with hindbrain expression $(\mathrm{HB}+, 64$ motifs in 112 $\mathrm{kb})$ compared with those with no hindbrain annotation (HB-, 85 in $238 \mathrm{~kb}$ ) (chi-square $\mathrm{p}=0.0042$ ). We then focused upon those sub-regions within EB enhancers that align directly with CNEs. Within these deeply conserved regions, there was more than two-fold enrichment for the stringent Pbx-Hox motif (30 occurrences in $24990 \mathrm{bp}$ of $\mathrm{HB}+$ elements compared with 32 occurrences in $60341 \mathrm{bp}$ of $\mathrm{HB}$ - elements; $\mathrm{p}=0.001$ ). Importantly, this enrichment demonstrates that $\mathrm{Pbx}-\mathrm{Hox}$ motifs in ancient CNEs show a correlation with hindbrain reporter expression. We also analysed a smaller dataset from the cneBrowser [21] that contains evolutionarily conserved enhancers associated with genes expressed in forebrain and hindbrain during zebrafish development. Although only 18 of 146 enhancers are annotated as hindbrain positive, 7 out of a total of 17 identified KR motifs reside in hindbrain positive enhancers $\left(\mathrm{p}=3 \times 10^{-4}\right)($ Table 1$)$. 


\section{Gnathostome alignments}

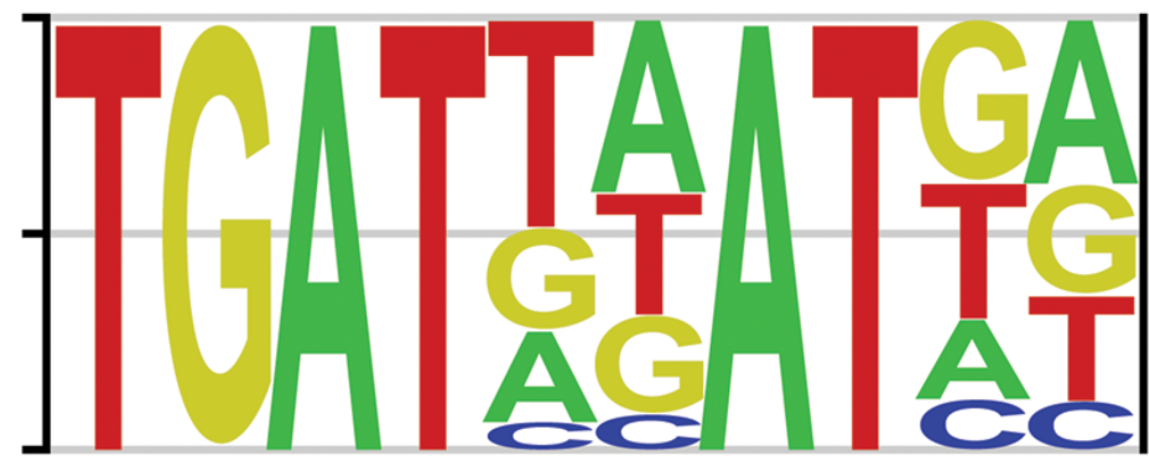

Human CNEs

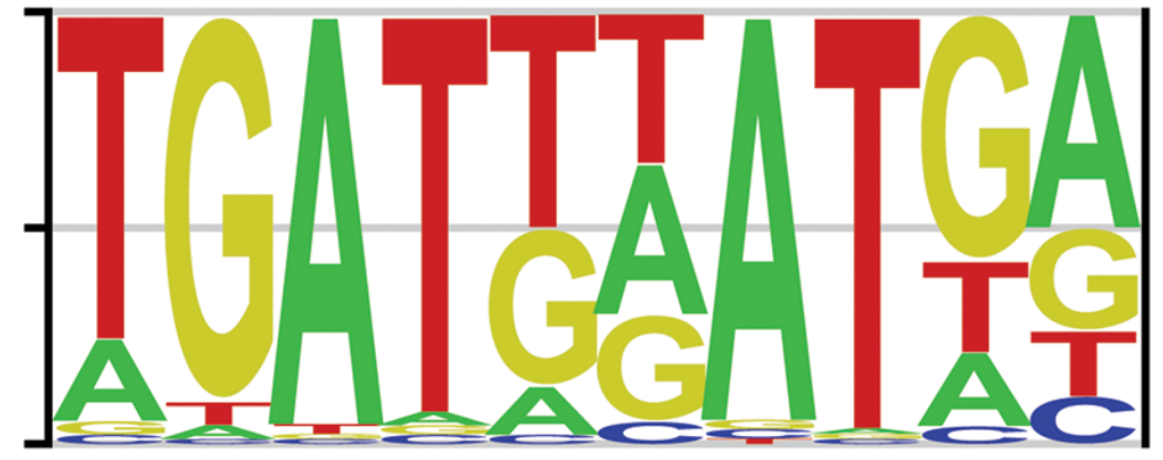

Literature

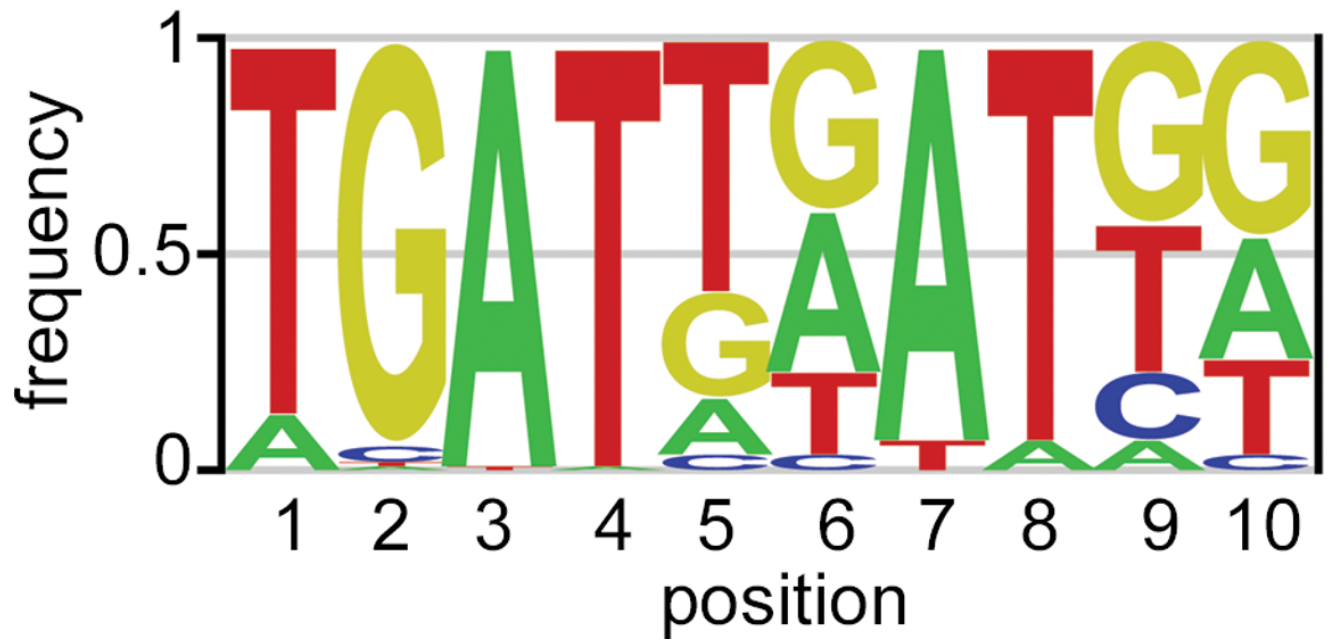

Figure $3 \mathrm{Pbx}$-hox motifs in CNEs strongly resemble verified PBX-HOX binding-sites. Position frequency logos generated from Gnathostome alignments (based on 712 conserved human TGATNNAT motifs in 4529 CNE alignments), Human CNEs (generated from the CONDOR CNE set using Cis-finder [41]) and from previous studies [36] (Literature). The relative base frequencies at positions 5 and 6, and 9 and 10, in CNEs, are in good agreement with known functional Pbx-Hox binding sites, supporting a strong KR consensus. 
Table 1 Frequency of KR motifs, compared to shuffled versions, in different test sets

\begin{tabular}{|c|c|c|c|c|c|c|c|}
\hline Motif & $\begin{array}{l}\text { CONDOR } \\
\text { CNEs (from } \\
\text { [57]) }\end{array}$ & $\begin{array}{l}\text { Human:Shark } \\
\text { CNEs (from 13] }\end{array}$ & $\begin{array}{l}\text { VISTA EB } \\
\text { (all) [43] }\end{array}$ & $\begin{array}{l}\text { VISTA EB set HB/BA/ } \\
\text { CN+ve (from [43]) }\end{array}$ & $\begin{array}{l}\text { VISTA EB set HB } \\
\text { +ve (from [43]) }\end{array}$ & $\begin{array}{l}\text { Zebrafish } \\
\text { CBset all } \\
\text { [21] }\end{array}$ & $\begin{array}{l}\text { Zebrafish CB set HB } \\
\text { +ve (from [21]) }\end{array}$ \\
\hline TGATNNATKR & 562 & 666 & 609 & 161 & 131 & 17 & 7 \\
\hline TGTANNATKR & 171 & 188 & 388 & 65 & 52 & 12 & 3 \\
\hline GTATNNATKR & 150 & 168 & 279 & 54 & 39 & 9 & 2 \\
\hline GTTANNATKR & 150 & 178 & 325 & 79 & 65 & 8 & 2 \\
\hline TTGANNATKR & 200 & 245 & 447 & 80 & 64 & 9 & 1 \\
\hline TTAGNNATKR & 167 & 238 & 398 & 74 & 55 & 7 & 0 \\
\hline ATGTNNATKR & 259 & 297 & 452 & 86 & 72 & 20 & 1 \\
\hline ATTGNNATKR & 233 & 297 & 436 & 74 & 61 & 20 & 2 \\
\hline AGTTNNATKR & 215 & 254 & 431 & 85 & 68 & 9 & 0 \\
\hline TAGTNNATKR & 147 & 154 & 297 & 54 & 42 & 10 & 4 \\
\hline TATGNNATKR & 176 & 198 & 365 & 74 & 60 & 10 & 3 \\
\hline GATTNNATKR & 274 & 315 & 419 & 97 & 74 & 11 & 0 \\
\hline TGATNNTAKR & 106 & 143 & 314 & 65 & 50 & 6 & 1 \\
\hline TGTANNTAKR & 142 & 151 & 421 & 82 & 60 & 14 & 1 \\
\hline GTATNNTAKR & 59 & 73 & 195 & 41 & 34 & 1 & 0 \\
\hline GTTANNTAKR & 105 & 108 & 253 & 50 & 33 & 5 & 0 \\
\hline TTGANNTAKR & 162 & 205 & 385 & 72 & 62 & 10 & 0 \\
\hline TTAGNNTAKR & 73 & 97 & 235 & 41 & 31 & 0 & 0 \\
\hline ATGTNNTAKR & 103 & 124 & 376 & 64 & 55 & 3 & 0 \\
\hline ATTGNNTAKR & 136 & 158 & 305 & 57 & 42 & 6 & 1 \\
\hline AGTTNNTAKR & 85 & 121 & 320 & 64 & 50 & 5 & 1 \\
\hline TAGTNNTAKR & 66 & 69 & 198 & 37 & 27 & 1 & 0 \\
\hline TATGNNTAKR & 84 & 94 & 345 & 80 & 62 & 5 & 1 \\
\hline GATTNNTAKR & 144 & 177 & 292 & 58 & 42 & 2 & 0 \\
\hline mean & 165.38 & 196.58 & 353.54 & 70.58 & 55.46 & 8.33 & 1.25 \\
\hline S.D & 102.75 & 122.06 & 93.73 & 24.74 & 20.92 & 5.52 & 1.67 \\
\hline $\begin{array}{l}\text { z-score for } \\
\text { pbxhox }\end{array}$ & 3.86 & 3.84 & 2.72 & 3.65 & 3.61 & 1.57 & 3.43 \\
\hline p-value & $5.68 \mathrm{E}-05$ & $6.16 \mathrm{E}-05$ & 3.30E-03 & 1.00E-04 & 2.00E-04 & $\mathrm{N} / \mathrm{S}$ & $3.00 \mathrm{E}-04$ \\
\hline
\end{tabular}

Enrichment analysis for Pbx-Hox KR motifs, relative to shuffled versions (retaining G+C content for each binding site), within different sets of CNEs. CNEs from the VISTA enhancer browser (EB) and zebrafish cneBrowser (CB) sets have also been grouped according to annotated expression in the hindbrain (HB), branchial arches $(B A)$ or cranial nerves $(\mathrm{CN})$. All sequences are human except the Zebrafish cneBrowser set. N/S = not significant

\section{CNEs containing Pbx-Hox motifs are associated with} genes that have roles in A-P patterning of the hindbrain and head

We have examined the distribution of Pbx-Hox motifs across CNEs of different genes, to ask whether genes with the highest enrichment of Pbx-Hox motifs in their CNEs have roles in hindbrain or pharyngeal arch patterning (Table 2 and Additional file 6). In keeping with the common use of auto-regulation in gene-regulatory networks [45], we find the CNEs of the HOXD cluster and the Hox co-factors, $P B X 3$ and MEIS2, to be amongst those with the highest number of these motifs. Many of the other genes with the highest density of Pbx-Hox motifs in their CNEs have characterised roles in anterior-posterior (A-P) head patterning and show segment specific patterns of expression during development. For instance, the ZNF503/703 (Nlz1 and Nlz2) zinc-finger proteins are essential for specification of rhombomere 4 in zebrafish $[46,47]$. The orphan nuclear receptor genes $N R 2 F 1 / 2$ (COUP-TF1/2) are negative transcriptional regulators involved in the retinoic acid signalling pathway, which has a key role in A-P patterning of the hindbrain and pharyngeal arches [48]. The members of the teashirt protein family (TSHZ1, 2 and 3) show segment-specific hindbrain expression [49], $T s h z 1$ being essential for segmentally restricted gene expression in the hindbrain and pharyngeal arches of frog and mouse [50,51].

There is good agreement between the genes highlighted by our in-silico binding-site search and by microarray screens for downstream targets of hoxb1 in rhombomere 4 of zebrafish [52] and mouse [53]. Specifically, the expression levels of $z n f 503$, tshz2, evi1, zic4, shox, and meis 2.1 are decreased upon knock-down of 


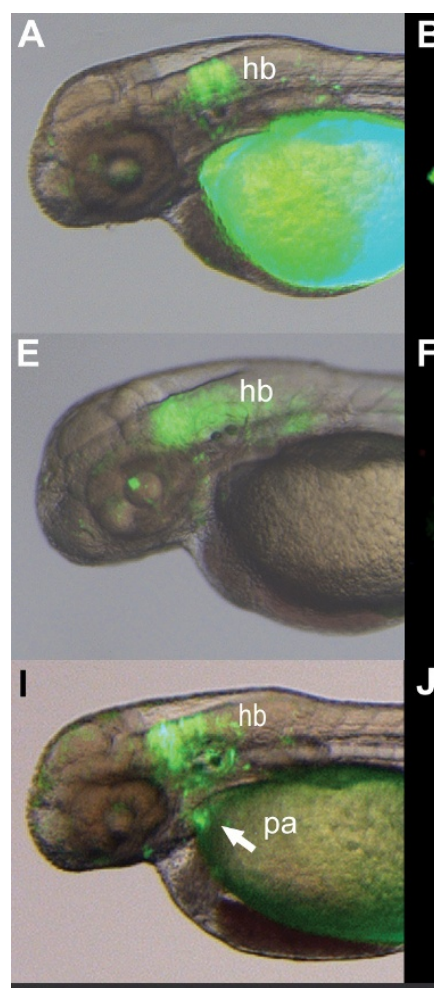

M

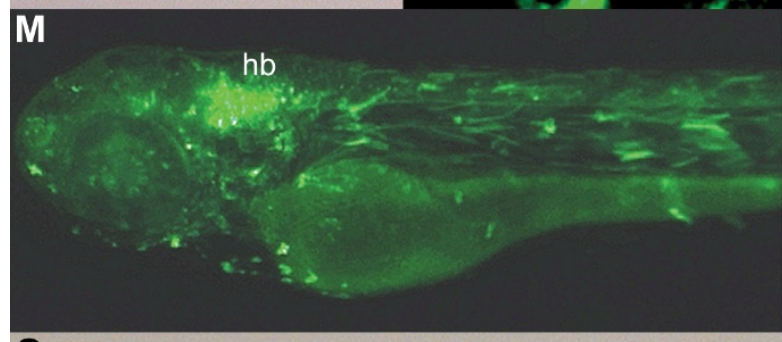

0

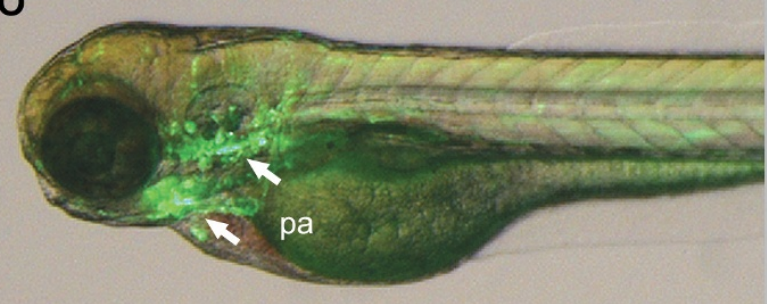

Q

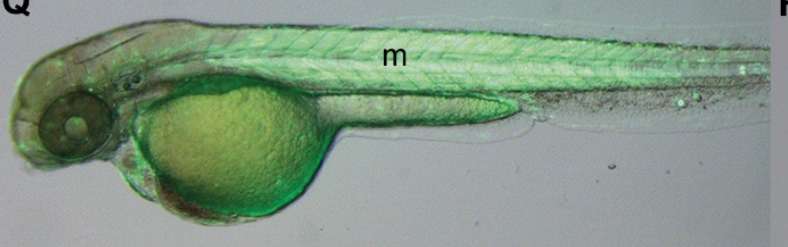

B

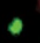

r3

$\mathbf{F}$
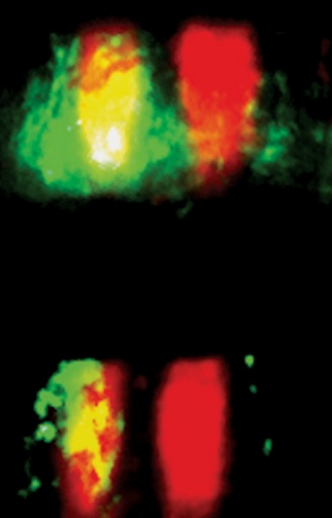

4
$\mathbf{N}$

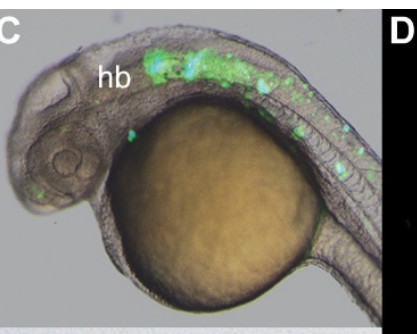

H

D

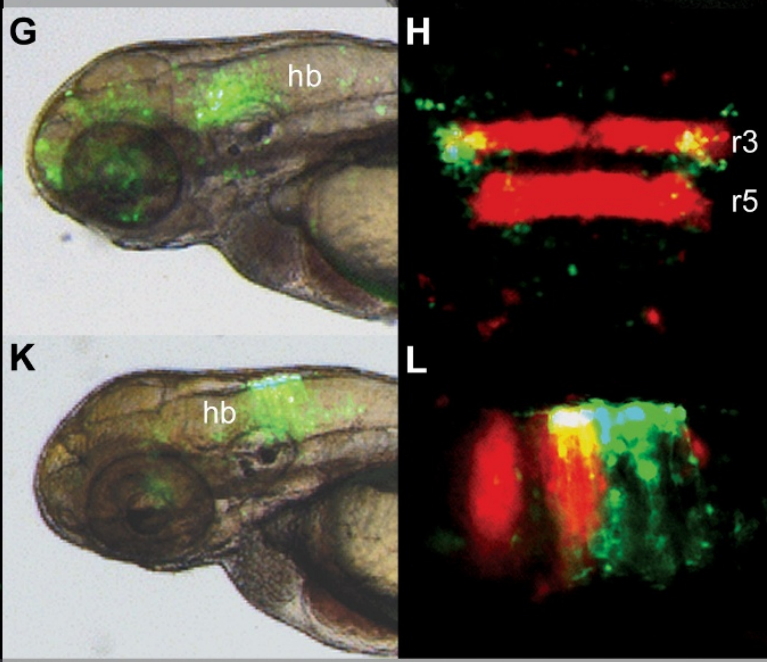

N

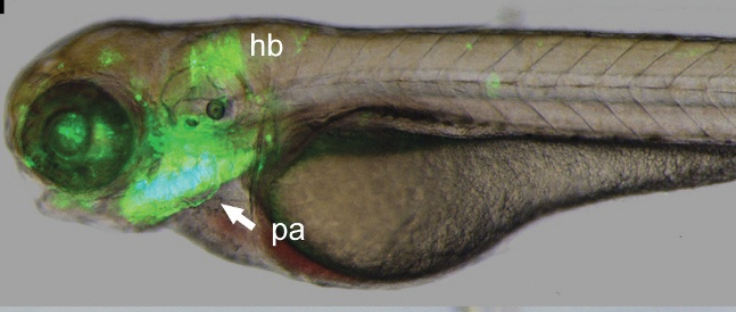

$\mathbf{P}$

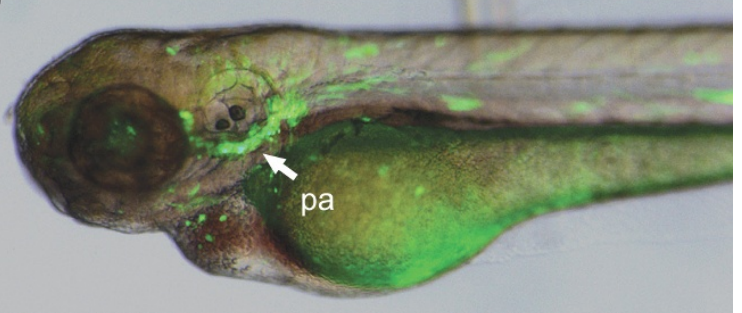

$\mathbf{R}$

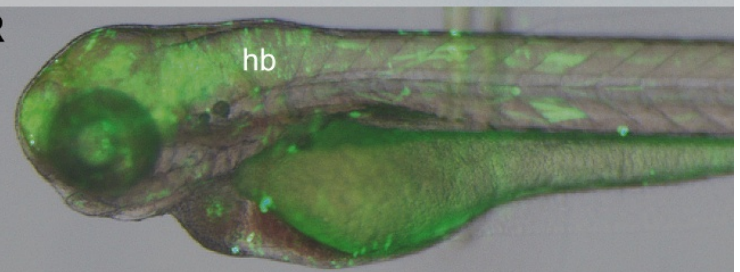

Figure $4 \mathrm{Pbx}-\mathrm{Hox}$ motifs correlate with segment-specific hindbrain and pharyngeal arch reporter expression. A-R, zebrafish elements from the lamprey $(\mathbf{A}-\mathbf{J}, \mathbf{M}, \mathbf{O}, \mathbf{Q})$ and jawed vertebrate $(\mathbf{K}, \mathbf{L}, \mathbf{N}, \mathbf{P}, \mathbf{R})$ CNE sets drive GFP expression in the hindbrain and pharyngeal arches. Elements: Evi1_40224 (A, B), Tshz3_43509 (C, D), NR2F2_27254 (E, F), Pax2_217 (G, dorsal view: H), ZNF503_32799 (I, J), Nkx6-1_4281 (K, L), Tshz3_24804 (M), Pax9_2099 (N), TshZ3_24805-6 (0), FoxP1_886 (P), Tshz3_24807 (Q), BCL11A_2554 (R). Expression in the hindbrain is often restricted to certain rhombomeres, as shown by comparison with r3r5 RFP expression (B, D, F, H, J, L). Tshz3_24807 drives expression in the trunk musculature (Q). Elements show temporal variation in reporter expression, expressing most strongly at 24-30hpf (C, D), 48-54hpf (A, B, E, F, I, J, Q) or 72-78hpf (G, H, K, L, M, N, O, P, R). hb: hindbrain; pa: pharyngeal arches; m: muscle. 
Table 2 Frequency of KR motifs in CNEs at different gene loci

\begin{tabular}{|c|c|c|c|c|c|c|c|}
\hline GENE & $\begin{array}{l}\text { \# KR motifs in test } \\
\text { set }\end{array}$ & $\begin{array}{l}\text { Length of CNE seq for locus } \\
(\mathrm{kb})\end{array}$ & $\begin{array}{l}\text { \#hits per } \\
\mathrm{kb}\end{array}$ & $\begin{array}{l}\text { \# hits in control set } \\
\text { (mean) }\end{array}$ & $\begin{array}{l}\text { standard } \\
\text { deviation }\end{array}$ & $\begin{array}{l}\text { z- } \\
\text { score }\end{array}$ & $\mathrm{p}$-value \\
\hline ZNF503 & 36 & 27.781 & 1.30 & 3.18 & 1.76 & 18.62 & $\begin{array}{l}0.00 \mathrm{E} \\
+00\end{array}$ \\
\hline TSHZ3 & 30 & 23.323 & 1.29 & 3.09 & 1.77 & 15.23 & $\begin{array}{l}0.00 \mathrm{E} \\
+00\end{array}$ \\
\hline IRX5 & 27 & 37.059 & 0.73 & 5.39 & 2.33 & 9.29 & $\begin{array}{l}0.00 \mathrm{E} \\
+00\end{array}$ \\
\hline IRX2 & 21 & 23.981 & 0.88 & 3.10 & 1.80 & 9.95 & $\begin{array}{l}0.00 \mathrm{E} \\
+00\end{array}$ \\
\hline TSHZ1 & 16 & 10.351 & 1.55 & 1.63 & 1.32 & 10.93 & $\begin{array}{l}0.00 \mathrm{E} \\
+00\end{array}$ \\
\hline PBX3 & 16 & 17.886 & 0.89 & 1.89 & 1.35 & 10.44 & $\begin{array}{l}0.00 \mathrm{E} \\
+00\end{array}$ \\
\hline HOXD9 & 16 & 17.77 & 0.90 & 2.19 & 1.44 & 9.59 & $\begin{array}{l}0.00 E \\
+00\end{array}$ \\
\hline NR2F2 & 16 & 18.99 & 0.84 & 2.52 & 1.59 & 8.49 & $\begin{array}{l}0.00 E \\
+00\end{array}$ \\
\hline NR2F1 & 16 & 25.655 & 0.62 & 3.72 & 1.84 & 6.67 & $2.53 \mathrm{E}-11$ \\
\hline MEIS2 & 16 & 24.553 & 0.65 & 3.42 & 1.91 & 6.59 & $4.49 \mathrm{E}-11$ \\
\hline $\mathrm{ZFHX1B}$ & 13 & 23.275 & 0.56 & 3.13 & 1.72 & 5.73 & $9.86 \mathrm{E}-09$ \\
\hline SALL3 & 12 & 11.405 & 1.05 & 1.43 & 1.21 & 8.76 & $\begin{array}{l}0.00 \mathrm{E} \\
+00\end{array}$ \\
\hline FOXP1 & 12 & 15.857 & 0.76 & 1.73 & 1.24 & 8.25 & $2.22 \mathrm{E}-16$ \\
\hline MAF & 11 & 7.334 & 1.50 & 1.15 & 1.10 & 8.95 & $\begin{array}{l}0.00 E \\
+00\end{array}$ \\
\hline NKX6-1 & 10 & 6.853 & 1.46 & 0.82 & 0.92 & 9.94 & $\begin{array}{l}0.00 E \\
+00\end{array}$ \\
\hline
\end{tabular}

Details are shown for the 15 gene loci from the CONDOR CNE set with the highest number of Pbx-Hox KR motifs in their CNEs, showing enrichment relative to shuffled CNE sets (Methods and Additional File 6). For each gene locus, the number of Pbx-Hox KR motifs in the associated CNEs is given. The number of PbxHox KR motifs per kb of CNE sequence for each locus (column 4) is calculated by dividing the number of Pbx-Hox KR motifs in the CNEs of that locus (column 2) by the total combined length of the CNEs in that locus (column 2). Control sets were generated by zero order Markov shuffling of CNEs at each locus in 1000 randomisations (Methods). Some gene loci also contain other genes besides the one after which they are named, for instance the IRX5 locus contains Irx3, Irx5 and Irx6.

HoxB1 in zebrafish, with $Z n f 503, N k x 6-1, A t b f 1$ and Mab21l2 down-regulated in HoxB1-/- mouse embryos. Accordingly, the CNEs around each of these genes are enriched in Pbx-Hox motifs (Table 2 and Additional File 6). Thus, both microarray datasets are consistent with our prediction that Pbx-Hox motifs in CNEs represent direct regulatory links between Hox genes and their targets during development.

\section{Discussion}

\section{Discovery of $\mathrm{Pbx}-\mathrm{Hox}$ motif enrichment is a further step} toward de-coding CNEs

Despite a pervasive assumption that CNEs bind transcription factors in order to elicit gene activation, there is, perhaps surprisingly, very little direct evidence to confirm this. We sought to identify TFBS motifs in CNEs through phylogenetic footprinting, reasoning that the relatively high divergence of lamprey CNEs would highlight important motifs. The utility of this approach is confirmed by our identification of conserved Pbx-Hox and Meis TFBS motifs in CNEs. The enrichment of the Pbx-Hox TFBS motif in the jawed vertebrate CNE set reveals this motif to be a regulatory signature that is utilised by a large proportion of highly conserved cis-regulatory elements (the 6, 693 CONDOR CNEs contain 562 KR motifs and 1416 TGATNNAT motifs). Whilst enriched motifs identified in mammalian conserved elements include a few that show partial overlap with variants of the Pbx-Hox consensus motif [23,24], the link between those enriched motifs and Hox factors had not been made, and their strong enrichment in more ancient CNEs had not been characterised. This enrichment agrees with the crucial, conserved roles of Hox factors in development of the vertebrate body plan. Indeed, the association of these motifs with hindbrain and pharyngeal arch enhancer function is in keeping with the characterised roles of $\mathrm{Pbx}, \mathrm{Hox}$ and Meis factors in patterning these domains.

Despite the crucial roles of Hox factors in patterning the vertebrate embryo, relatively few downstream target genes, other than the hox genes themselves, have been identified. Our data suggests that Pbx-Hox motifs in CNEs can identify such targets. The striking manner in which Pbx-Hox and Meis TFBS motifs are highlighted 
as conserved sequence blocks in multiple alignments, especially when lamprey sequences are included, leads us to predict that this footprinting approach will be useful for further deciphering the regulatory code within vertebrate enhancers. In combination, our in-silico and functional analyses form an important link between well characterised cis-regulatory motifs and a large proportion of relatively uncharacterised ancient CNEs, helping to better place these elements within a developmental context. This represents a significant further step in systematically de-coding the enhancers responsible for development of the vertebrate body plan and highlights the utility of the lamprey as a model system for investigating vertebrate gene regulation.

The diversity of expression patterns driven by our tested elements suggests that Pbx-Hox TFBSs are just one component of a complex cis-regulatory logic encoded within these enhancers. Whilst responding to A-P patterning cues by interacting with particular Hox factors through Pbx-Hox TFBSs, these elements concomitantly determine the tissues in which they are active (e.g. hindbrain vs pharyngeal arch) and limit the expression patterns dorso-ventrally, medio-laterally and temporally. An example of this is the CNE Pax2_403, which drives reporter expression that is restricted to a ventrolateral population of neurons in $\mathrm{r} 2-3$ of the hindbrain (Figure 4). Furthermore, whilst some of our functionally characterised CNEs drive reporter expression in domains with sharp boundaries that are co-incident with rhombomere boundaries -similar to that of previously characterised $\mathrm{Pbx}-\mathrm{Hox}$ regulated elements - this is not the case for all of them. This could be due to the $\mathrm{Pbx}-\mathrm{Hox}$ input establishing a competence for the enhancer to drive expression within particular rhombomeres, which is further restricted to specific sub-domains within the rhombomeres by the influence of other regulatory inputs to the enhancer. This would result in expression domains that do not encompass the whole area of expression of the regulating Hox factor. It is likely that the reason why many previously characterised $\mathrm{Pbx}-\mathrm{Hox}$ regulated elements show expression domains across whole rhombomeres and with tight boundaries co-incident with rhombomere boundaries is that the majority of these elements are regulating Hox factors, and thus setting up or maintaining the rhombomerespecific Hox expression patterns. Many of the elements described in this study may be acting downstream of this Hox network, utilising these AP patterning cues along with other cues to further pattern the hindbrain. The tissue specificity of these enhancers, as well as the restriction of expression to specific domains and time points, is presumably due to other factors acting as specifiers by binding to nearby TFBSs. Identifying these specifiers and characterising their TFBSs, as well as the nature of their interactions with Hox factors, are key tasks toward understanding the cis-regulatory logic underlying vertebrate development. The set of putative Hox-responsive cis-regulatory elements identified in this study provides a powerful resource that will facilitate efforts toward this end.

Our expression data from the mutated versions of zebrafish CNE 3299 suggests that the multiple Pbx-Hox and Meis sites predicted in this enhancer may interact with each other, to co-operatively modulate and restrict reporter expression. The two clusters of $\mathrm{Pbx}-\mathrm{Hox}$ and Meis motifs do not contribute equally to the expression driven by this enhancer in the hindbrain and pharyngeal arch neural crest. The second Pbx-Hox and Meis motif cluster appears to be necessary for the general function of this enhancer, as its mutation results in the loss of reporter expression in both hindbrain and neural crest. In contrast, the first $\mathrm{Pbx}-\mathrm{Hox}$ and Meis motif cluster appears to be necessary (but not sufficient) for neural crest expression, but not for hindbrain expression. Conversely, it appears to restrict the hindbrain expression, as reporter expression is seen more anteriorly when this cluster is mutated. This is reminiscent of interactions between Pbx-Hox and Meis/Prep binding sites within a Hoxb1 enhancer, which direct expression of this gene to $\mathrm{r} 4$ the hindbrain in mouse and chick [54]. In that case, it was found that the formation of a Pbx-Hox-Meis/Prep ternary complex on $\mathrm{Pbx}-\mathrm{Hox}$ and Meis sites within this enhancer could be restricted by the binding of a Pbx1Prep1 heterodimer to a nearby site, thus limiting the expression driven by this enhancer to $\mathrm{r} 4$. This highlights the complexity of the regulatory interactions between transcription factors that are likely to bind to CNEs, a complexity that could well underlie their high sequence constraint.

\section{A potential role for CNEs in the evolution of vertebrate head patterning}

A strength of identifying conserved cis-regulatory elements is that they can provide compelling evidence for conserved GRNs. Our reporter assay data from zebrafish and lamprey embryos clearly demonstrate functional conservation of enhancers shared between the most distantly related extant vertebrate lineages. We deduce that all vertebrates share aspects of a GRN for hindbrain patterning, downstream of nested Hox expression. As the sea lamprey is from a vertebrate lineage that diverged prior to the evolution of many jawed vertebrate innovations, such as paired appendages and jaws [55], we predict that the lamprey reporter assay will be a crucial tool for investigating the gene regulatory changes involved in vertebrate evolution.

Without detailed knowledge of the function or mechanism of action of CNEs it has been difficult to 
derive scenarios of how they evolved and became fixed in vertebrate genomes. The findings from our in silico and functional analyses, coupled with previous characterisation of $\mathrm{Pbx}-\mathrm{Hox}$ and Meis transcription-factor complexes, enable us to propose a hypothesis regarding the role of a large number of CNEs in vertebrate evolution. Recognising the same TFBS motifs in worms, flies and vertebrates, the Pbx, Hox and Meis factors are part of an ancient regulatory language shared across bilaterians $[17,36,37,56,57]$. Nevertheless, none of the CNEs containing these motifs are identifiable in invertebrate genomes, leading us to speculate that many of these elements may have arisen in the vertebrate lineage. Accordingly, our functional data suggest that many of these CNEs have roles in patterning an elaborate head and brain - key vertebrate innovations [54]. We hypothesise that the fundamental role of head patterning in vertebrates led to the functional conservation of these elements and that their reliance upon the precise organisation of TFBSs necessitated their strict sequence conservation. The mechanisms through which new cisregulatory elements arise in the genome are still largely unresolved. In this case, the finding that simple PbxHox sites are sufficient to drive robust and specific, but modifiable, expression [17] hints that these particular TFBSs may pioneer new cis-regulatory elements, functioning as one of the fundamental seeds from which many CNEs were able to grow.

\section{Conclusions}

The finding that vertebrate CNEs are highly enriched for $\mathrm{Pbx}$-Hox binding-site motifs represents a further step toward de-coding ancient vertebrate enhancers. Coupled with our experimental data, this enables a large proportion of these elements to be more firmly placed into a developmental context and reveals ancient gene regulatory network interactions for hindbrain and head patterning that were present in ancestral vertebrates. Finally, our findings lead us to hypothesise that the evolution of many of these CNEs contributed to the elaboration of the vertebrate hindbrain and the branchial region of the head.

\section{Methods}

\section{Identification of CNEs}

6, 693 non-redundant human CNEs (average length 116 bp) were retrieved from the CONDOR database [43] at http://condor.nimr.mrc.ac.uk (Additional File 7). We used these to search lamprey sequence reads available from the NCBI trace server at http://www.ncbi.nlm.nih. gov/Traces/trace.cgi with sensitive parameters (-W 7 -q -1 -e $5 \mathrm{e}-4$ ) as described previously [28]. The lamprey trace sequences were searched because they represent a greater coverage of the lamprey genome than the publicly available draft genome assembly, which consists of many short contigs and thus provides little advantage with regard to identification of conserved syntenic regions. Lamprey sequences satisfying the initial parametric threshold were further analysed for contamination, and those with $>90 \%$ homology to human or chicken across the whole read (i.e. extending outside the evolutionarily conserved region in other vertebrates) were removed.

\section{Alignments}

The sequences of human, fugu and most zebrafish CNEs were retrieved from the CONDOR database [57]. Additional zebrafish CNEs that were not previously included in the CONDOR database due to absence from earlier assemblies were identified using BLAST against a more recent zebrafish genome assembly (Zv8 release 58 ). Sequences in each alignment were clipped to the same size to prevent unaligned edges. To align the sequences we used ClustalW version 1.83. These alignments formed the lamprey CNE set (comprised of alignments of CNEs from human, fugu, zebrafish and lamprey) and the gnathostome CNE set (containing alignments of CNEs from human, fugu and zebrafish). As a control, for each CNE we also generated 1000 multiple alignments by randomly shuffling the columns of each alignment using the seqboot implementation in Phylip version 3.67. The sequences of lamprey and zebrafish CNEs in these datasets are given in Additional File 8 and Additional File 9 respectively. The sequences of CNEs from the EB and cneBrowser datasets are given in Additional File 10 and Additional File 11 respectively.

\section{Scanning CNEs and control sets for Pbx-Hox motifs}

We searched for Pbx-Hox motifs in two different types of datasets. Firstly, in multiple sequence alignments of CNEs from the CONDOR CNE database (the lamprey and gnathostome CNE sets). Secondly, in datasets consisting of sequences from just one species (the EB CNEs, shark CNEs, human elements from the CONDOR CNE set, cneBrowser CNEs). The two different types of datasets required different types of control to test for Pbx-Hox motif enrichment. For the alignment sets, we generated control sets of shuffled alignments. To find evolutionarily conserved Pbx-hox motifs (TGATNNAT and TGATNNATKR) we employed the software Cis-Finder [42] on our two alignment sets and their respective shuffled alignment controls. A motif match was only considered if it matched all aligned species and occurred at the exact same aligned position. For the single species sequence sets we generated shuffled motifs, based upon the KR motif, as a control to search across the same sets. In parallel we also employed a de-novo motif finding strategy implemented 
in Cis-Finder on the CONDOR CNE set. It scans a set of DNA sequences for over-represented position frequency matrices (PFMs), clusters these and then estimates significance using the false discovery rate [42]. The TGATNNAT and TGATNNATKR motif occurances in the CONDOR CNE set are detailed in Additional File 12 and Additional File 13.

To characterise the frequency of Pbx-Hox KR motifs in different gene loci, we used the CONDOR CNE set, in which CNEs are grouped according to gene locus as specified in the CONDOR database [57]. For each gene locus, we counted the frequency of Pbx-Hox KR motifs in the associated CNEs and compared this to the average frequency in 1000 sets of randomised versions of CNEs from that locus. A markov chain model of order zero was used to generate shuffled sequences. To model DNA sequences, 4 states (A, C, G, T) and 4 transitions were used. Transition probabilities were retrieved from the CNE set by calculating the relative frequencies of the bases.

\section{Measuring relative enrichment of $\mathrm{Pbx}-\mathrm{Hox}$ motifs}

To measure the enrichment, we compared the occurrence of $\mathrm{Pbx}$-Hox motifs in a test set against shuffled versions (Table 1), calculated mean and standard deviation and generated $\mathrm{z}$-scores. The $\mathrm{z}$-scores were then transformed into $\mathrm{p}$-values under a normal distribution model. We also counted Pbx-Hox occurrence and shuffled versions in a number of control regions and across the whole human genome (Additional File 4).

\section{Overlap with other evolutionarily conserved 'enhancer' sets}

There is inevitably some overlap between the different sets of evolutionarily conserved sequences. 482/1307 EB human sequences overlap 994 CONDOR CNEs, covering a total of 146226 bases (7.4\% of the EB sequence; $18.8 \%$ of CNE sequence). 1632 human sequences identified through comparison with Callorhinchus milii [13] overlap 2172 CONDOR CNEs, covering a total of 271260 bases (26.5\% of the Callorhinchus dataset; $34.9 \%$ of CNEs). Finally, 69/146 zebrafish cneBrowser sequences overlap 83 CONDOR CNEs, covering a total of 11496 bases (20.5\% of the cneBrowser sequence; $1.5 \%$ of CNE sequence).

\section{Zebrafish transgenesis}

CNEs were amplified from zebrafish and lamprey genomic DNA by PCR, sub-cloned into the Pcr8/GW/TOPO vector (Invitrogen) and then into a Tol 2 construct (pGW_cfosEGFP) [29,58,59], using the Gateway LR Clonase II enzyme (Invitrogen). The Tol2 reporter assay was performed as described previously [29]. Transient transgenic zebrafish embryos were screened for GFP expression at 24-30hpf, 48-54hpf and 72-78hpf using a Leica M165FC microscope and photographs taken with a Leica DFC310FX camera. Expression patterns were deemed consistent when found in $>20 \%$ of founders, consistent with previous studies $[25,60]$.

\section{CNE Mutagenesis}

Mutations in zebrafish CNE 3299 were introduced by PCR from genomic DNA with primers containing the desired mutations either through conventional PCR (for sub1) or megaprimer PCR (for sub2) [61]. Mutated CNE PCR products were then cloned for zebrafish transgenesis as described above.

\section{Lamprey transgenesis}

The transgenesis protocol was based upon that developed in Xenopus [62]. Lamprey CNEs were amplified from genomic DNA and cloned into the cFos-I-sceI-EGFP plasmid, which contains the mouse cFos minimal promoter and EGFP coding sequence flanked by I-sceI restriction sites. Plasmids were extracted using the EndoFree Plasmid Maxi Kit (Qiagen) and eluted with water through QIAQuick columns (Qiagen). Fresh restriction digests (20 $\mu$ l containing $400 \mathrm{ng}$ plasmid, 15 units I-SceI enzyme (NEB), $1 \times$ I-SceI buffer + BSA, digested for 40 minutes at $37^{\circ} \mathrm{C}$ ) were micro-injected into 5-6hpf lamprey embryos using a Pico-Spritzer with drop volume of 2-3 nl. Lamprey husbandry was performed as described previously [63]. Embryos were screened for GFP expression between embryonic days 7-16. Typical survival rates ranged from $20-50 \%$ of injected embryos. The promoter alone drives highly mosaic background expression in the ectoderm in roughly $50 \%$ of surviving embryos. Enhancer-specific expression was seen in approximately $10 \%$ of surviving embryos.

\section{Additional material}

Additional file 1: Genomic sequences used to generate MLAGAN alignment. The genomic sequences of human, fugu, zebrafish and lamprey used to generate the MLAGAN alignment in this study.

Additional file 2: CNEs functionally tested for this study. The sequences of the CNEs of zebrafish ( $d r)$ and lamprey $(\mathrm{pm})$ that were tested by reporter assay in zebrafish embryos for this study.

Additional file 3: The frequency of Pbx-Hox motifs in different test sets. A table describing the frequency of Pbx-Hox motifs in different test sets, compared with 1000 randomised controls.

Additional file 4: The frequency of KR motifs in different control sets. A table listing the frequency of KR motifs, compared to shuffled versions, in different control sets.

Additional file 5: The frequency of Meis motifs in Human CNEs. A table listing the frequency of Meis motifs, compared to shuffled versions in human CONDOR CNEs.

Additional file 6: The frequency of KR motifs in CNEs at different gene loci. A table listing the frequency of KR motifs in CNES at different gene loci. 
Additional file 7: The CONDOR CNE set. 6693 non-redundant human CNE sequences, shared between human and fugu, retrieved from the CONDOR database.

Additional file 8: Lamprey CNEs. Lamprey sequences of 246 CNES shared between jawed vertebrates and lamprey (from the lamprey CNE alignment set)

Additional file 9: Zebrafish CNEs. Zebrafish sequences of 4259 CNEs shared between human, fugu and zebrafish (from the gnathostome CNE alignment set).

Additional file 10: CNEs from the VISTA Enhancer Browser. 1307 human sequences from the VISTA Enhancer Browser http://enhancer.lbl. gov/.

Additional file 11: CNEs from the cneBrowser. 146 functionally tested zebrafish sequences from the cneBrowser http://bioinformatics.bc.edu/ chuanglab/cneBrowser/\#home.

Additional file 12: TGATNNAT motif hits on the human CONDOR CNEs. Hits of the TGATNNAT motif on the human CONDOR CNEs listed by CNE with details of the start and finish of the motif within the CNE.

Additional file 13: TGATNNATKR motif hits on the human CONDOR CNEs. Hits of the TGATNNATKR motif on the human CONDOR CNES listed by CNE with details of the start and finish of the motif within the CNE.

\section{Abbreviations}

CNE: conserved non-coding element; TFBS: transcription factor binding site; GRN: gene regulatory network; EB: enhancer browser (dataset); A-P: anteriorposterior.

\section{Acknowledgements}

We are indebted to Andrew McCallion and Koichi Kawakami for supplying us with the pGW_cfosEGFP construct, and Reinhard Koester for the r3r5 transgenic zebrafish line. We thank Stefan Pauls and Debbie Goode for helpful suggestions on the manuscript. This work was funded by MRC Project Grant 72504 to GE and NIH Grant DE017911 to MB. HJP was funded by a QMUL PhD studentship.

\section{Author details}

'Division of Systems Biology, MRC National Institute for Medical Research, The Ridgeway, Mill Hill, London NW7 1AA, UK. ²Weatherall Institute of Molecular Medicine, University of Oxford, John Radcliffe Hospital, Oxford OX3 9DS, UK. ${ }^{3}$ Division of Biology, California Institute of Technology, Pasadena, CA 91125, USA.

\section{Authors' contributions}

Conceived and designed the experiments: HJP and GE. Performed the experiments: HJP. Conceived and designed bio-informatic analyses: PP, HJP and GE. Performed bio-informatic analyses: PP and GE. Analysed the data: HJP, PP and GE. Supplied materials, reagents and lamprey expertise: MB and TS-S. Wrote the paper: HJP, PP and GE. All authors read and approved the final manuscript.

Received: 7 October 2011 Accepted: 30 December 2011 Published: 30 December 2011

\section{References}

1. Lettice LA, Heaney SJ, Purdie LA, Li L, de Beer P, Oostra BA, Goode D, Elgar G, Hill RE, de Graaff E: A long-range Shh enhancer regulates expression in the developing limb and fin and is associated with preaxial polydactyly. Hum Mol Genet 2003, 12:1725-35.

2. Jeong Y, Leskow FC, El-Jaick K, Roessler E, Muenke M, Yocum A, Dubourg C, Li X, Geng X, Oliver G, Epstein DJ: Regulation of a remote Shh forebrain enhancer by the Six3 homeoprotein. Nat Genet 2008, 40:1348-53.

3. Visel A, Rubin EM, Pennacchio LA: Genomic views of distant-acting enhancers. Nature 2009, 461:199-205.

4. Siepel A, Bejerano G, Pedersen JS, Hinrichs AS, Hou M, Rosenbloom K, Clawson H, Spieth J, Hillier LW, Richards S, Weinstock GM, Wilson RK,
Gibbs RA, Kent WJ, Miller W, Haussler D: Evolutionarily conserved elements in vertebrate, insect, worm, and yeast genomes. Genome Res 2005, 15:1034-50.

5. Aparicio S, Morrison A, Gould A, Gilthorpe J, Chaudhuri C, Rigby P, Krumlauf $R$, Brenner $S$ : Detecting conserved regulatory elements with the model genome of the Japanese puffer fish, Fugu rubripes. Proc Natl Acad Sci USA 1995, 92:1684-8.

6. Woolfe A, Goodson M, Goode DK, Snell P, McEwen GK, Vavouri T, Smith SF, North P, Callaway H, Kelly K, Walter K, Abnizova I, Gilks W, Edwards YJ, Cooke JE, Elgar G: Highly conserved non-coding sequences are associated with vertebrate development. PLoS Biol 2005, 3:e7.

7. Pennacchio LA, Ahituv N, Moses AM, Prabhakar S, Nobrega MA, Shoukry M, Minovitsky S, Dubchak I, Holt A, Lewis KD, Plajzer-Frick I, Akiyama J, De Val S, Afzal V, Black BL, Couronne O, Eisen MB, Visel A, Rubin EM: In vivo enhancer analysis of human conserved non-coding sequences. Nature 2006, 444:499-502.

8. Zeitlinger J, Zinzen RP, Stark A, Kellis M, Zhang H, Young RA, Levine M: Whole-genome ChIP-chip analysis of Dorsal, Twist, and Snail suggests integration of diverse patterning processes in the Drosophila embryo. Genes Dev 2007, 21:385-90.

9. Johnson DS, Mortazavi A, Myers RM, Wold B: Genome-wide mapping of in vivo protein-DNA interactions. Science 2007, 316:1497-502

10. Visel A, Blow MJ, Li Z, Zhang T, Akiyama JA, Holt A, Plajzer-Frick I, Shoukry M, Wright C, Chen F, Afzal V, Ren B, Rubin EM, Pennacchio LA: ChIP-seq accurately predicts tissue-specific activity of enhancers. Nature 2009, 457:854-8.

11. Bejerano G, Pheasant M, Makunin I, Stephen S, Kent WJ, Mattick JS, Haussler D: Ultraconserved elements in the human genome. Science 2004, 304:1321-5.

12. Sandelin A, Bailey P, Bruce $S$, Engström PG, Klos JM, Wasserman WW, Ericson J, Lenhard B: Arrays of ultraconserved non-coding regions span the loci of key developmental genes in vertebrate genomes. BMC Genomics 2004, 5:99.

13. Venkatesh B, Kirkness EF, Loh YH, Halpern AL, Lee AP, Johnson J, Dandona N, Viswanathan LD, Tay A, Venter JC, Strausberg RL, Brenner S: Ancient noncoding elements conserved in the human genome. Science 2006, 314:1892.

14. de la Calle-Mustienes E, Feijóo CG, Manzanares M, Tena JJ, RodríguezSeguel E, Letizia A, Allende ML, Gómez-Skarmeta JL: A functional survey of the enhancer activity of conserved non-coding sequences from vertebrate Iroquois cluster gene deserts. Genome Res 2005, 15:1061-72

15. Ragvin A, Moro E, Fredman D, Navratilova P, Drivenes $\varnothing$, Engström PG, Alonso ME, de la Calle Mustienes E, Gómez Skarmeta JL, Tavares MJ, Casares F, Manzanares M, van Heyningen V, Molven A, Njølstad PR, Argenton F, Lenhard B, Becker TS: Long-range gene regulation links genomic type 2 diabetes and obesity risk regions to HHEX, SOX4, and IRX3. Proc Natl Acad Sci USA 2010, 107:775-80

16. Holland LZ, Albalat R, Azumi K, Benito-Gutiérrez E, Blow MJ, BronnerFraser M, Brunet F, Butts T, Candiani S, Dishaw L, Ferrier DE, GarciaFernàndez J, Gibson-Brown JJ, Gissi C, Godzik A, Hallböök F, Hirose D, Hosomichi K, Ikuta T, Inoko H, Kasahara M, Kasamatsu J, Kawashima T, Kimura A, Kobayashi M, Kozmik Z, Kubokawa K, Laudet V, Litman GW, McHardy AC, et al: The amphioxus genome illustrates vertebrate origins and cephalochordate biology. Genome Res 2008, 18:1100-1111.

17. Pöpperl H, Bienz M, Studer M, Chan SK, Aparicio S, Brenner S, Mann RS, Krumlauf R: Segmental expression of Hoxb-1 is controlled by a highly conserved autoregulatory loop dependent upon exd/pbx. Cell 1995, 81:1031-1042.

18. Ferretti $E$, Marshall $H$, Pöpperl $H$, Maconochie M, Krumlauf R, Blasi F: Segmental expression of Hoxb2 in $r 4$ requires two separate sites that integrate cooperative interactions between Prep1, Pbx and Hox proteins. Development 2000, 127:155-66.

19. Kurokawa D, Sakurai Y, Inoue A, Nakayama R, Takasaki N, Suda Y, Miyake T, Amemiya $C T$, Aizawa $S$ : Evolutionary constraint on Otx2 neuroectoderm enhancers - deep conservation from skate to mouse and unique divergence in teleost. Proc Nat Acad Sci USA 2006, 103:19350-5.

20. Bailey PJ, Klos JM, Andersson E, Karlén M, Källström M, Ponjavic J, Muhr J, Lenhard B, Sandelin A, Ericson J: A global genomic transcriptional code associated with CNS-expressed genes. Exp Cell Res 2006, 312:3108-19. 
21. Li Q, Ritter D, Yang N, Dong Z, Li H, Chuang JH, Guo S: A systematic approach to identify functional motifs within vertebrate developmental enhancers. Dev Biol 2010, 337:484-495.

22. Xie X, Lu J, Kulbokas EJ, Golub TR, Mootha V, Lindblad-Toh K, Lander ES, Kellis M: Systematic discovery of regulatory motifs in human promoters and $3^{\prime}$ UTRs by comparison of several mammals. Nature 2005, 434:338-345.

23. Xie X, Mikkelsen TS, Gnirke A, Lindblad-Toh K, Kellis M, Lander ES: Systematic discovery of regulatory motifs in conserved regions of the human genome, including thousands of CTCF insulator sites. Proc Natl Acad Sci USA 2007, 104:7145-7150.

24. Minovitsky S, Stegmaier P, Kel A, Kondrashov AS, Dubchak I: Short sequence motifs, overrepresented in mammalian conserved non-coding sequences. BMC Genomics 2007, 8:378.

25. Narlikar L, Sakabe NJ, Blanski AA, Arimura FE, Westlund JM, Nobrega MA, Ovcharenko I: Genome-wide discovery of human heart enhancers. Genome Res 2010, 20:381-92.

26. Jaeger SA, Chan ET, Berger MF, Stottmann R, Hughes TR, Bulyk ML: Conservation and regulatory associations of a wide affinity range of mouse transcription factor binding sites. Genomics 2010, 95:185-95.

27. Blair JE, Hedges SB: Molecular phylogeny and divergence times of deuterostome animals. Mol Biol Evol 2005, 22:2275-84.

28. McEwen GK, Goode DK, Parker HJ, Woolfe A, Callaway H, Elgar G: Early evolution of conserved regulatory sequences associated with development in vertebrates. PLoS Genet 2009, 5:e10000762.

29. Fisher S, Grice EA, Vinton RM, Bessling SL, Urasaki A, Kawakami K, McCallion AS: Evaluating the biological relevance of putative enhancers using Tol2 transposon-mediated transgenesis in zebrafish. Nat Protoc 2006, 1:1297-305.

30. Zerucha T, Prince VE: Cloning and developmental expression of a zebrafish meis2 homeobox gene. Mech Dev 2001, 102:247-50.

31. Biemar F, Devos N, Martial JA, Driever W, Peers B: Cloning and expression of the TALE superclass homeobox Meis2 gene during zebrafish embryonic development. Mech Dev 2001, 109:427-31.

32. Waskiewicz AJ, Rikhof HA, Hernandez RE, Moens CB: Zebrafish Meis functions to stabilize $\mathrm{Pbx}$ proteins and regulate hindbrain patterning. Development 2001, 128:4139-51.

33. Choe SK, Vlachakis N, Sagerström CG: Meis family proteins are required for hindbrain development in the zebrafish. Development 2002, 129:585-95.

34. Distel M, Wullimann MF, Köster RW: Optimised Gal4 genetics for permanent gene expression mapping in zebrafish. Proc Natl Acad Sci USA 2009, 106:13365-70.

35. Jacobs Y, Schnabel CA, Cleary ML: Trimeric association of Hox and TALE homeodomain proteins mediates Hoxb2 hindbrain enhancer activity. Mol Cell Biol 1999, 19:5134-42.

36. Maconochie MK, Nonchev S, Studer M, Chan SK, Pöpperl H, Sham MH Mann RS, Krumlauf R: Cross-regulation in the mouse HoxB complex: the expression of Hoxb2 in rhombomere 4 is regulated by Hoxb1. Genes Dev 1997, 11(14):1885-95.

37. Mann RS, Lelli KM, Joshi R: Hox specificity: unique roles for cofactors and collaborators. Curr Top Dev Biol 2009, 88:63-101.

38. Chang CP, Brocchieri L, Shen WF, Largman C, Cleary ML: Pbx modulation of Hox homeodomain amino-terminal arms establishes different DNAbinding specificities across the Hox locus. Mol Cell Biol 1996, 16:1734-45.

39. Chang $C P$, Jacobs $Y$, Nakamura $T$, Jenkins NA, Copeland NG: Meis proteins are major in vivo DNA binding partners for wild-type but not chimeric Pbx proteins. Mol Cell Biol 1997, 17:5679-87.

40. Chan SK, Mann RS: A structural model for a homeotic proteinextradenticle-DNA complex accounts for the choice of HOX protein in the heterodimer. Proc Natl Acad Sci USA 1996, 93:5223-8.

41. Joshi R, Passner JM, Rohs R, Jain R, Sosinsky A, Crickmore MA, Jacob V, Aggarwal AK, Honig B, Mann RS: Functional specificity of a Hox protein mediated by the recognition of minor groove structure. Cell 2007, 131:530-43.

42. Sharov AA, Ko MSH: Exhaustive search for over-represented DNA sequence motifs with CisFinder. DNA Res 2009, 16:261-73.

43. Woolfe A, Goode DK, Cooke J, Callaway H, Smith S, Snell P, McEwen GK, Elgar G: CONDOR: a database resource of developmentally associated conserved non-coding elements. BMC Dev Biol 2007, 7:100.
44. Visel A, Minovitsky S, Dubchak I, Pennacchio LA: VISTA Enhancer Browsera database of tissue-specific human enhancers. Nucleic Acids Res 2007, 35: D88-92.

45. Davidson EH: The Regulatory Genome: Gene Regulatory Networks in Development and Evolution. Academic Press, San Diego; 2006.

46. Hoyle J, Tang YP, Wiellette EL, Wardle FC, Sive H: nlz gene family is required for hindbrain patterning in the zebrafish. Dev Dyn 2004, 229:835-46.

47. Runko AP, Sagerström CG: Nlz belongs to a family of zinc-fingercontaining repressors and controls segmental gene expression in the zebrafish hindbrain. Dev Biol 2003, 262:254-67.

48. Pereira FA, Tsai MJ, Tsai SY: COUP-TF orphan nuclear receptors in development and differention. Cell Mol Life Sci 2000, 57:1388-98.

49. Santos JS, Fonseca NA, Vieira CP, Vieira J, Casares F: Phylogeny of the Teashirt-related Zinc Finger (tshz) gene family and analysis of the developmental expression of tshz2 and tshz3b in the zebrafish. Dev Dyn 2010, 239:1010-18

50. Koebernick K, Kashef J, Pieler T, Wedlich D: Xenopus Teashirt1 regulates posterior identity in brain and cranial neural crest. Dev Biol 2006, 298:312-26.

51. Coré N, Caubit X, Metchat A, Boned A, Djabali M, Fasano L: Tshz1 is required for axial skeleton, soft palate and middle ear development in mice. Dev Biol 2007, 308:407-420.

52. Rohrschneider MR, Elsen GE, Prince VE: Zebrafish Hoxb1a regulates multiple downstream genes including prickle1b. Dev Biol 2007, 309:358-72.

53. Tvrdik P, Capecchi MR: Reversal of Hox1 gene subfunctionalisation in the mouse. Dev Cell 2006, 11:239-50.

54. Ferretti E, Cambronero F, Tümpel S, Longobardi E, Wiedemann LM, Blasi F, Krumlauf R: Hoxb1 enhancer and control of rhombomere 4 expression: complex interplay between PREP1-PBX1-HOXB1 binding sites. Mol Cell Biol 2005, 25:8541-52.

55. Shimeld SM, Holland PW: Vertebrate innovations. Proc Natl Acad Sci USA 2000, 97:4449-52

56. Streit A, Kohler R, Marty T, Belfiore M, Takacs-Vellai K: Conserved regulation of the Caenorhabditis elegans labial/Hox1 gene ceh-13. Dev Biol 2002, 242:96-108.

57. Ryoo HD, Marty T, Casares F, Affolter M, Mann RS: Regulation of Hox target genes by a DNA bound Homothorax/Hox/Extradenticle complex. Development 1999, 126:5137-48.

58. Urasaki A, Morvan G, Kawakami K: Functional dissection of the Tol2 transposable element identified the minimal cis-sequence and a highly repetitive sequence in the subterminal region essential for transposition. Genetics 2006, 174:639-49.

59. Kawakami K, Takeda H, Kawakami N, Kobayashi M, Matsuda N, Mishina M: A transposon-mediated gene trap approach identifies developmentally regulated genes in zebrafish. Developmental Cell 2004, 7:133-44.

60. McGaughey DM, Stine ZE, Huynh JL, Vinton RM, McCallion AS: Asymmetrical distribution of non-conserved regulatory sequences at PHOX2B is reflected at the ENCODE loci and illuminates a possible genome-wide trend. BMC Genomics 2009, 10:8.

61. Barik S: Megaprimer PCR. Methods Mol Biol 2002, 192:189-96.

62. Ogino H, McConnell WB, Grainger RM: High-throughput transgenesis in Xenopus using I-Scel meganuclease. Nat Protoc 2006, 1:1703-10.

63. Nikitina N, Bronner-Fraser M, Sauka-Spengler T: Culturing lamprey embryos. Cold Spring Harb Protoc 2009, doi:10.1101/pdb.prot5122.

doi:10.1186/1471-2164-12-637

Cite this article as: Parker et al: Ancient Pbx-Hox signatures define hundreds of vertebrate developmental enhancers. BMC Genomics 2011 12:637. 